\title{
Bivariate pointing movements on large touch screens: Investigating the validity of a refined Fitts' Law
}

\author{
Jennifer Bützler", Sebastian Vetter, Nicole Jochems and Christopher M. Schlick \\ Chair and Institute of Industrial Engineering and Ergonomics of RWTH Aachen University, Germany
}

\begin{abstract}
On the basis of three empirical studies Fitts' Law was refined for bivariate pointing tasks on large touch screens. In the first study different target width parameters were investigated. The second study considered the effect of the motion angle. Based on the results of the two studies a refined model for movement time in human-computer interaction was formulated. A third study, which is described here in detail, concerns the validation of the refined model. For the validation study 20 subjects had to execute a bivariate pointing task on a large touch screen. In the experimental task 250 rectangular target objects were displayed at a randomly chosen position on the screen covering a broad range of ID values (ID= $[1.01 ; 4.88])$. Compared to existing refinements of Fitts' Law, the new model shows highest predictive validity. A promising field of application of the model is the ergonomic design and evaluation of project management software. By using the refined model, software designers can calculate a priori the appropriate angular position and the size of buttons, menus or icons.
\end{abstract}

Keywords: Fitts' Law, bivariate pointing task, movement time, human factors of software-systems

\footnotetext{
*Corresponding author. E-mail: j.buetzler@iaw.rwth-aachen.de
} 


\section{Introduction}

Fitts' Law [4] is one of the most important quantitative models in the field of human-computer interaction (HCI). For goal-directed pointing movements Fitts' Law states a linear relationship between movement time (MT) and the difficulty of the task $I D$ (Index of Difficulty):

$$
M T=a+b \cdot I D \quad I D=\log _{\mathrm{s}}\left(\frac{\mathrm{a} a}{m_{1}}\right)
$$

The $I D$ of a movement is defined by the distance between start and target object, the amplitude of the movement $(A)$, and the size of the target as the horizontal target width $\left(W_{h}\right)$. The coefficients $a$ and $b$ are determined empirically by linear regression. By adapting the equation above one can use Fitts' Law to determine "optimal" target sizes and target positions in terms of movement time for information input.

In Fitts' original study the angle between start and target object was $0^{\circ}$ (movements to the right) or $180^{\circ}$ (movements to the left) and the target size $W_{h}$ was determined by the horizontal target width. Accordingly, the definition of the target size is univariate and the experimental task is one-dimensional. However in human-computer interaction one has to deal with two-dimensional movements. Thus, to use Fitts' Law as a predictive model in the field of HCI one has to consider two factors: The influence of the motion angle and the definition of the target width. The question of how to take account of two-dimensional pointing movements and rectangular, bivariate target objects was investigated and validated in three studies. The consolidated analyses and findings are repeated in the following section.

\section{Literature review}

\subsection{The target width in bivariate pointing tasks}

MacKenzie \& Buxton [7] analyzed different target width parameters in a bivariate pointing task with a mouse. In order to define the target size of rectangular target objects they investigated three different approach angles $0^{\circ}$ (movement to the right), $45^{\circ}$ (diagonal movements) and $90^{\circ}$ (vertical movements). Five target width models were compared in the study: The horizontal target width $\left(W_{h}\right)$, target height plus target width $\left(W_{h+w}\right)$, object area $\left(W_{h^{*}}\right)$, target width in the direction of motion $W^{\prime}$ (Eq. 2) and a model which considers the shortest length of the sides, the $W_{\text {min }}$ model (Eq. 3).

$$
\begin{aligned}
& \mathrm{MT}=a \mid b \log \left(\frac{A}{W^{\prime}} 1\right) \\
& \mathrm{MT}=\mathrm{a}+b \log :\left(\frac{A}{\mathrm{M}_{-}}+1\right)
\end{aligned}
$$

MacKenzie \& Buxton found the best fit between model and data for the $W_{\text {min }}$ model $\left(\mathrm{R}^{2}=0.950\right)$, followed by the $W^{\prime}$ model which determines the target width in the direction of motion $\left(\mathrm{R}^{2}=0.933\right)$. Although MacKenzie \& Buxton [7] found better fitting for $W_{\min }$, the parameter $W^{\prime}$ was established in the ISO standard [5]. Nonetheless, the $W_{\min }$ model is the de facto standard in most experimental and practical studies.

Accot and Zhai [1] investigated Fitts' Law in a pointing task with a motion angle of $0^{\circ}$ (movements to the right) and $180^{\circ}$ (movements to the left) with rectangular target objects of different side lengths ratios. The results show a complex interaction of target width and target height. Accot and Zhai [1] suggest the following model:

$$
\mathrm{MT}=\mathrm{a}+\mathrm{b} \log =\left(\sqrt{\left(\frac{A}{w}\right)^{2}+\eta\left(\frac{A}{n}\right)^{2}+1}\right)
$$

$W$ refers to the horizontal target width and $H$ to the vertical target height. The constant $\eta$ can be chosen within the range of $1 / 7$ and $1 / 3$. A validation of the model yielded a high coefficient of determination of $\mathrm{R}^{2}=0.994$.

\subsection{The angle in bivariate pointing tasks}

As mentioned above, MacKenzie and Buxton [7] arranged rectangular target objects at different angles between start and target objects $\left(0^{\circ}, 45^{\circ}, 90^{\circ}\right)$ to investigate the effects of the angle. The results regarding the motion angle showed that movement time was lowest when start and target object were arranged horizontally $\left(0^{\circ}\right)$. In the study of Whisenand and Emurian [10] the angle between start and target object was varied in eight steps $0^{\circ}, 45^{\circ}, 90^{\circ}, 135^{\circ}$, $180^{\circ}, 225^{\circ}, 270^{\circ}$ and $315^{\circ}$, whereas $0^{\circ}$ defines movements to the right and $180^{\circ}$ movements to the left. The pointing task was executed with a computer mouse. In accordance with MacKenzie and Buxton [7] the results showed that movement time was lowest for start and target objects that were arranged on a 
horizontal line $\left(0^{\circ}, 180^{\circ}\right)$. Iwase and Murata $[6,8]$ examined movement times using either a computer mouse or a touch panel. They conducted a pointing task with circular target objects for the same motion angles as Whisenand and Emurian used $\left(\alpha=0^{\circ}, 45^{\circ}\right.$, $\left.90^{\circ}, 135^{\circ}, 180^{\circ}, 225^{\circ}, 270^{\circ}, 315^{\circ}\right)$. For touch panels they found a periodical sinusoidal relationship between movement time and motion angle, whereas no angle effect was found for the pointing task with the mouse.

Appert et al. [2] investigated seven angles in a pointing task with a mouse $\left(\alpha=0^{\circ}, 30^{\circ}, 60^{\circ}, 90^{\circ}\right.$, $120^{\circ}, 150^{\circ}, 180^{\circ}$; for a better understanding and a consistent definition of the angle, the angle $\gamma$ which was used in the original study was transferred to $\alpha$ : $\gamma=90^{\circ}-\alpha$ ). The highest movement times were found for an angle $\alpha$ of $90^{\circ}$, the lowest movement times occurred for an angle $\alpha$ of $0^{\circ}$ and $180^{\circ}$. A model was derived from the observation and expanded by the angle. The model reached a high fit of $\mathrm{R}^{2}=0.960$ :

$$
\mathrm{MT}=\mathrm{a}+\mathrm{b} \log \mathrm{g}\left(\frac{A}{\mathrm{M}}+\frac{A}{\mathrm{H}}+0,6 \cos \left(9 \mathrm{C}^{\mathrm{a}}-\mathrm{a}\right) \frac{\mathrm{A}}{\min \mid \mathrm{W} h \mathrm{~h}}+1\right)
$$

Yang and $\mathrm{Xu}$ [11] compared different variants of Fitts' Law with regard to their goodness of fit. The $\mathrm{W}_{\text {min }}$ model (Eq. 3) yielded a coefficient of determination of $\mathrm{R}^{2}=0.924$. The model of Accot and Zhai [1] reaches a $\mathrm{R}^{2}=0.923$ (Eq. 4) and the model which was expanded by the angle $\alpha$ by Appert et al. [2] a $\mathrm{R}^{2}=0.916$ (Eq. 5). The target width in the direction of movement ( $\mathrm{W}^{\prime}$, Eq. 2) was not investigated. Yang and $\mathrm{Xu}$ developed an own ,Augmented Regular Configuration Model“", which fits the data best $\left(\mathrm{R}^{2}=0.955\right)$ :

$$
\mathrm{MT}=\mathrm{a}+b \log :\left(\frac{a}{\left(\frac{A}{w}\right)^{2}+\left(\frac{a}{b}\right)^{2}+1}\right)+\operatorname{clog} \alpha
$$

\subsection{Evaluation of the state of the art}

As initially mentioned, in $\mathrm{HCI}$ a typical pointing task is two-dimensional with regard to the principle direction of the movement and bivariate with regard to the target object.

MacKenzie and Buxton [7] presented rectangular target objects at different motion angles to obtain a variation of the target width in the direction of motion $\mathrm{W}^{\prime}$. However, the variation of the target width and the angle within one experiment can lead to the problem that the effects of the target width might be influenced by the angle. Likewise the effects of the angle might be confounded by effects of the target width. Whisenand and Emurian [10], Appert et al. [2] and Yang and $\mathrm{Xu}$ (2010) used rectangular target objects in their studies and arranged them at different angles. Therefore, the angle effects found in their studies possibly are confounded by the target width in the direction of motion that also varied for different angles. Furthermore, the often insufficient validation of the model has to be criticized. In most studies one data set is used for both, the derivation and the validation of a model. This procedure of testing the model against itself however is tautological (circular reasoning) and necessarily results in a high coefficient of determination.

\subsection{Previous work of the authors}

In order to refine Fitts' Law systematically regarding two-dimensional pointing movements and bivariate target objects two empirical studies with a large touch screen were conducted.

\subsubsection{Effect of target width in bivariate pointing tasks}

In the first study [9] the two most common target width definitions $W_{\min }$ and $W^{\prime}$ were analyzed in an empirical study with 15 right-handed subjects aged between 20 and 32 years. In the experimental task rectangular target objects were displayed at a constant angle of $0^{\circ}$ (movement to the right) and constant amplitude of $324.3 \mathrm{~mm}$. The target objects were rotated in $10^{\circ}$ steps $\left(0^{\circ}\right.$ to $\left.90^{\circ}\right)$ around their centre of gravity. Hereby, $W^{\prime}$ was varied systematically, whereas $W_{\text {min }}$ was kept constant. Based on the results of the study, the $W_{\min }$ model was rejected. Furthermore, significant effects of the target height perpendicular to the direction of motion $\left(H^{\prime}\right)$ were found. The best empirical fit was found for a model taking into account the target width in the direction of motion $\left(W^{\prime}\right)$ and the target height perpendicular to the direction of motion $\left(H^{\prime}\right)$ :

$$
M T=a+b \log :\left(\frac{a}{2}+1\right)+\operatorname{dog}:\left(\frac{a}{2}+1\right)
$$

The parameter $c$ was determined empirically by linear regression.

\subsubsection{Effect of angle in bivariate pointing tasks}

In the second study [3] the motion angle was investigated in a pointing task on a large scaled touch screen. 15 right-handed subjects aged between 21 and 36 years participated in the study. The subjects 
were divided by age into two groups with 15 persons each. The angle $\alpha$ between start and target object was varied systematically in $10^{\circ}$ steps between $0^{\circ}$ (movements to the right) and $180^{\circ}$ (movements to the left) for two amplitudes (200 mm, $400 \mathrm{~mm})$. To eliminate confounding influences of the target width, circular target objects were used. The results support Murata's and Iwase's findings [8], namely that movement time follows a sinus-shaped curve depending on the angle:

$$
M T=2+b \log \left(\frac{\pi}{w^{2}}+1\right)+o \sin (2 \alpha)
$$

In Figure 1 the empirical and modeled movement time data are depicted for the two amplitudes. This manifest model yields a high fitting with the experimental data, resulting in a $\mathrm{R}^{2}$ of 0.984 .

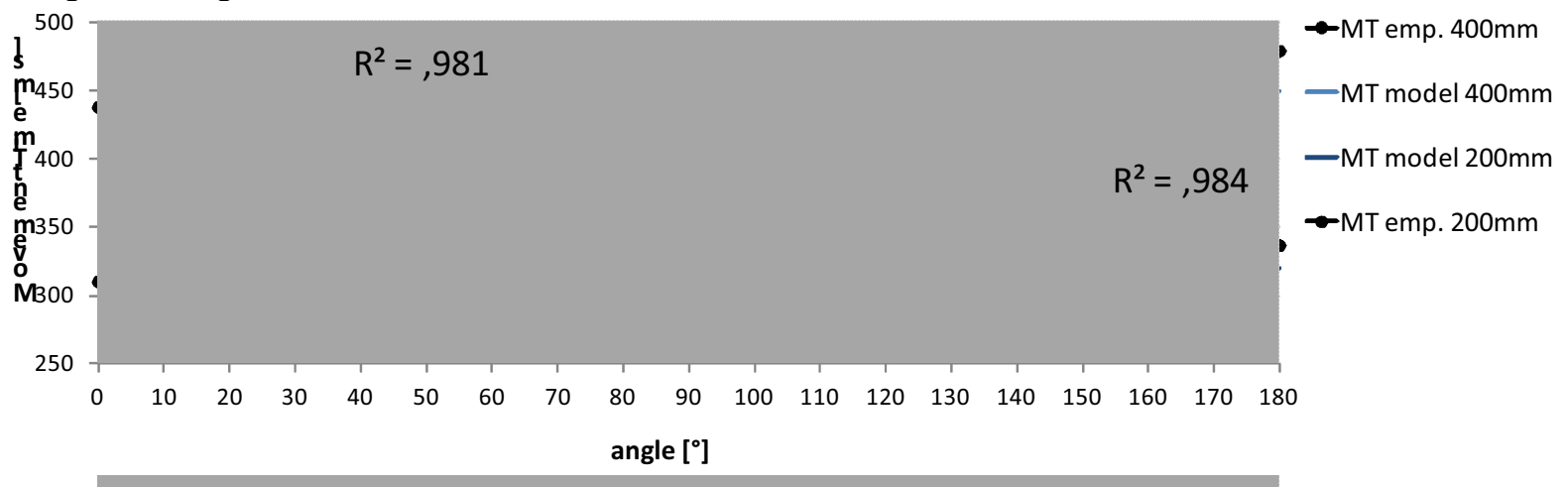

Fig. 1. Empirical and modeled movement time data

Based on the results of both studies a model for two-dimensional pointing and bivariate, rectangular target objects was formulated:

$$
\text { MT }=a+b \log :\left(\frac{A}{W}+1\right)+\operatorname{clog}\left(\frac{A}{E}+1\right)+d \sin (2 \alpha)
$$

The aim of the third study was to examine the predictive validity of the model from Eq. 9.

\section{Method}

\subsection{Subjects}

The sample consisted of 20 right-handed subjects, nine women and eleven men, aged between 21 and 35 years (M: 27 years, SD: 4.65 years).

\subsection{Apparatus}

The hardware used to measure the pointing movements was the DiamondTouch screen developed by Circletwelve Inc. The DiamondTouch screen is a tabletop device (projection area $865 \mathrm{~mm} \mathrm{x}$
$649 \mathrm{~mm}, 4: 3$ ratio) with a touch-sensitive surface of $1070 \mathrm{~mm}$ in diagonal. The images are projected from top via an LCD projector (1600 x 1200 pixels). Through capacitive coupling between a transmitter array embedded in the touch surface and separate receivers the subjects sit on, the attached control unit can distinguish multiple touch inputs.

\subsection{Procedure}

The pointing task was carried out with the DiamondTouch screen lying on a table with a height of $755 \mathrm{~mm}$. The subjects were seated on a chair in front of the screen. The experimenter demonstrated and supervised a sample target block to familiarize the subject with the task and the test environment. The subjects were instructed to point as fast and accurately as possible. The experimental task consisted of a typical pointing task which was carried out with the right index finger. Starting from a central home position $(\varnothing=20 \mathrm{~mm})$ at the bottom of the touch screen each subject had to point the same sequence of 250 rectangular target objects four times. The target objects were arranged randomly on the screen. To exclude extremely small or large target objects, boundaries were set for the horizontal and 
vertical target width of $\min =20 \mathrm{~mm}$ and $\max =60 \mathrm{~mm}$. The target geometry was chosen because in HCI, buttons, menu items and graphical user interface elements are mostly rectangular and possess a greater width than height as they usually contain text. Therefore, the horizontal target width $W_{h}$ was chosen to be higher or equal to the vertical target width $W_{v}$. The $I D$ of the pointing task is calculated according to Eq. 2. By the arrangement of the 250 target objects (Figure 2, left side) an $I D$ range of $[1.01 ; 4.88]$ was covered. To avoid an over- or underrepresentation of particular ID values, an approximate uniform frequency distribution was ensured (Figure 2, right side).
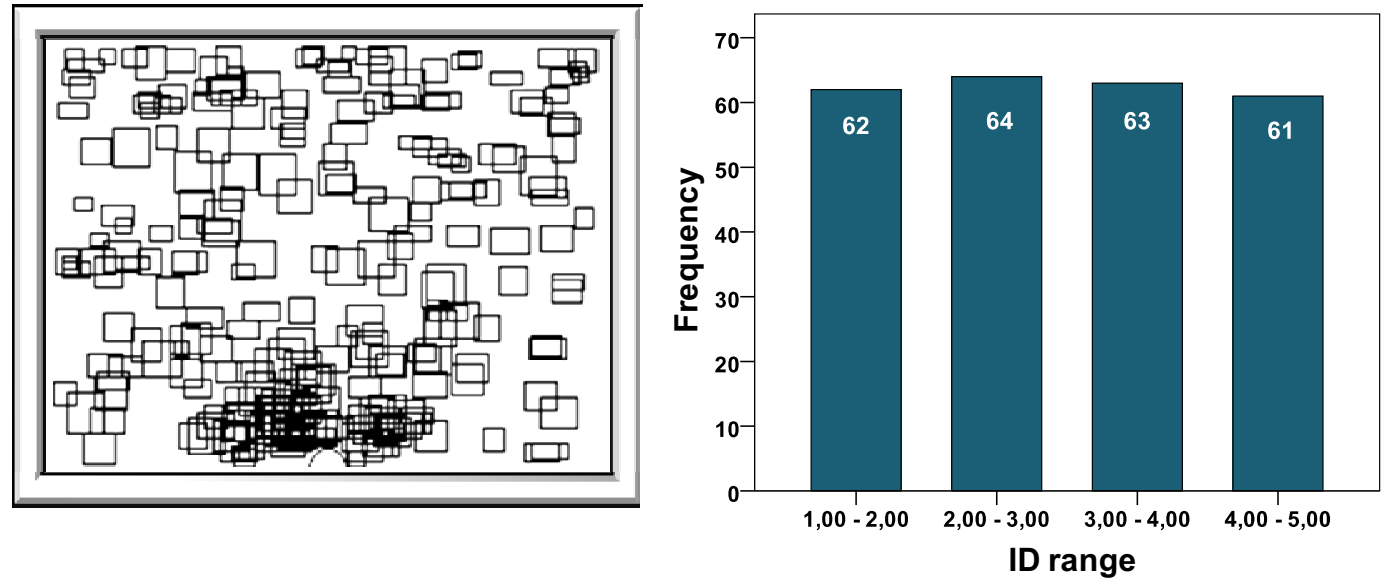

Fig. 2. Arrangement of the 250 target objects (left side) and frequency distribution of ID-ranges (right side).

\subsection{Model evaluation}

To model the movement time linear and non-linear regression models were developed. To determine the goodness of fit of the corresponding model the coefficient of determination $\mathrm{R}^{2}$ was calculated.

\section{Results}

Figure 3 shows the fit between model and data of Fitts' Law calculated by Eq. 2 in the upper diagram
[7] and the enhanced Fitts' Law model of the authors (Eq. 9) in the lower diagram. The model of the authors reaches a higher coefficient of determination with $\mathrm{R}^{2}=0.937$ than the model by MacKenzie and Buxton [7] $\left(\mathrm{R}^{2}=0.905\right)$. As shown in Figure 3, the model of the authors explains variability in movement time better, by also taking account of target height and motion angle. 

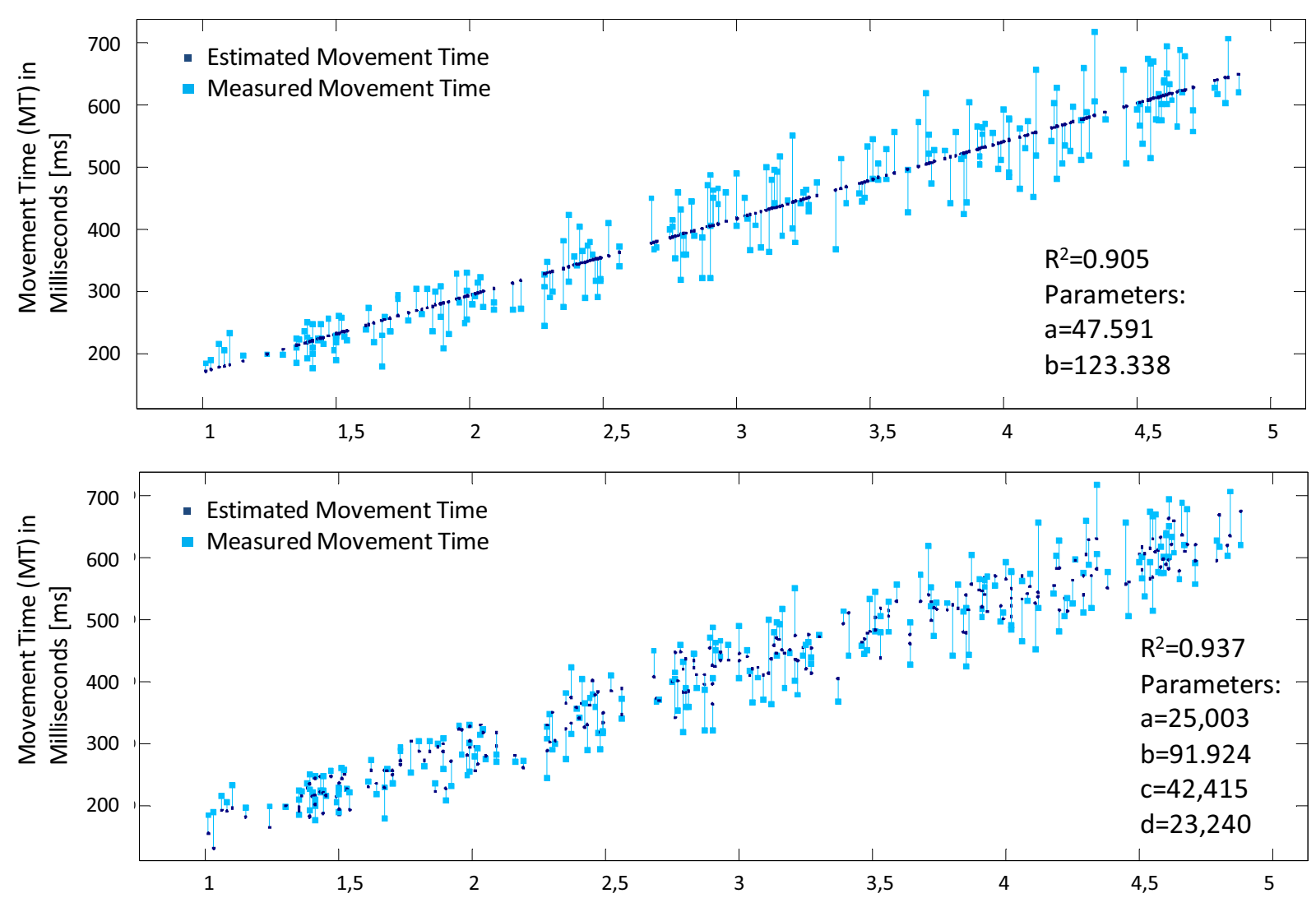

Fig. 3. Measured an predicted. The predictions were carried out on the basis of the model by MacKenzie and Buxton Eq. 2 (upper part) and the model of the authors Eq. 9 (lower part).

A comparison of the goodness of fit of all models (Eq. 1 to Eq. 9) that are considered in this paper can be found in table 1 .
All models yielded a fit of $\mathrm{R}^{2}>0.896$. The highest coefficient of determination of $\mathrm{R}^{2}=0.937$ was found for equation 9 , the self-developed model of the authors.

Table 1. Comparison of different Fitts' Law models (Eq. 1 - Eq. 9)

\begin{tabular}{|c|c|c|c|}
\hline Eq. no. & Authors & Equation of the model & $\mathbf{R}^{2}$ \\
\hline \multicolumn{4}{|c|}{ univariate target width } \\
\hline 1 & Fitts (1954) & $M T=a+b \log _{2}\left(\frac{a}{w^{2}}\right)$ & 0.898 \\
\hline \multicolumn{4}{|c|}{ bivariate target width } \\
\hline 2 & $\begin{array}{l}\text { Mackenzie (1992); } \\
\text { DIN EN ISO } 9241\end{array}$ & $M I=a+b \log :\left(\frac{A}{w^{2}}+1\right)$ & 0.905 \\
\hline 3 & $\begin{array}{l}\text { Mackenzie (1992); } \\
\text { de facto Standard }\end{array}$ & $M T=a+b \log :\left(\frac{a}{w-n}+z\right)$ & 0.896 \\
\hline 4 & $\begin{array}{l}\text { Accot \& Zhai } \\
(2003)\end{array}$ & $M T=a+b \log _{2}\left(\sqrt{\left(\frac{a}{w}\right)^{2}+\eta(a)+1}\right) ; \eta=0,24$ & 0.913 \\
\hline
\end{tabular}




\begin{tabular}{|c|c|c|c|}
\hline 7 & $\begin{array}{l}\text { Vetter \& Bützler } \\
\text { (2011) }\end{array}$ & $M T=a+b \log _{a}\left(\frac{a}{w}+1\right)+0 \log _{2}\left(\frac{a}{a}+1\right)$ & 0.922 \\
\hline \multicolumn{4}{|c|}{ bivariate target width and additional angle term } \\
\hline 5 & $\begin{array}{l}\text { Appert et al. } \\
(2008)\end{array}$ & $M T=a+b \log =\left(\frac{A}{w}+\frac{a}{g}+0,6 \cos \left(90^{\circ}-a\right) \frac{a}{\operatorname{man}\left(w_{i} n\right)}+1\right)$ & 0.924 \\
\hline 6 & Yang \& Xu (2010) & $M T=a+b \log _{2}\left(\frac{2}{2} \sqrt{\left(\frac{a}{2}\right)^{2}+\left(\frac{a}{a}\right)^{2}+1}\right)+\operatorname{clog}(\alpha)$ & 0.924 \\
\hline 9 & $\begin{array}{l}\text { Vetter \& Bützler } \\
\text { (2011) }\end{array}$ & $M T=a+b \log _{2}\left(\frac{A}{n}+1\right)+c \log _{2}\left(\frac{A}{\pi}+1\right)+a \sin (2 \alpha)$ & 0.937 \\
\hline
\end{tabular}

\section{Discussion}

On the basis of two empirical studies, the authors developed a refined model for time prediction of rapid aimed movements on large touch screens. According to the refined model, movement time is a function of task difficulty in and perpendicular to the direction of motion and the angular position of the target object. Within a third study the refined model was compared to existing models regarding their predictive validity. It was found that the refined model explains variability in movement time better than the other models with a high coefficient of determination $\mathrm{R}^{2}=0.937$. However, it has to be considered if the greater predictive validity justifies the additional computational effort. Regarding the predictive validity all models achieve a high value of $\mathrm{R}^{2}>0.896$, whereas the computational effort differs. The decision on the suitability of a particular model depends on the purpose of application. For example if one wants a first estimation about how changing the distance or target size presumably effects the movement time, Fitts' Law can be used with low computational effort in its original formulation [4] (Eq. 1). However, if detailed insights are necessary, the model of the authors (Eq. 9) provides a better prediction. A promising field of application for the proposed model is the ergonomic design of project management software. The model can be used to determine an ergonomic "optimal" angle for the arrangement of buttons and interaction elements in an early conceptual stage of software design. Furthermore, the model can be used a priori to calculate the best proportions of the side lengths for buttons and interaction fields depending on the position on the screen.

\section{References}

[1] J. Accot and S. Zhai, Refining Fitts' law models for bivariate pointing, in: Proc. Conference on Human Factors in Computing Systems, ACM CHI, Ft Lauderdale, USA, 2003, pp. 193200.

[2] C. Appert, O. Chapuis and M. Beaudouin-Lafon, Evaluation of Pointing Performance on Screen Edges, in Proc. ACM Advanced Visual Interfaces, Napoli, Italy. ACM Press, New York, 2008, pp. 119-126.

[3] J. Bützler, S. Vetter, N. Jochems and C.M. Schlick, Refinement of Fitts's Law for Large Touch Screens: AgeDifferentiated Analysis of the Motion Angle in Bivariate Pointing Tasks, in: Proc. Tenth International Symposium on Human Factors in Organizational Design and Management, Grahamstown, South Africa, 2011, pp. 93-99.

[4] P.M. Fitts, The information capacity of the human motor system in controlling the amplitude of movement, Journal of Experimental Psychology 47 (1954), 381-391.

[5] ISO, 2002. Reference Number: ISO 9241-9 (E), Ergonomic requirements for office work with visual display terminals (VDTs)-Part 9-Requirements for non-keyboard input devices (ISO 9241-9) (Vol. February 15, 2002): International Organization for Standardization.

[6] H. Iwase and A. Murata, Empirical study on improvement of usability for touch-panel for elderly - comparison of usability between touch-panel and mouse, in: Systems, Man, and Cybernetics, IEEE International Conference, 2002, pp. 252-257.

[7] I.S. MacKenzie and W. Buxton, Extending Fitts' law to twodimensional tasks, in: W. Bauersfeld, P. Bennett, J. Lynch, (Eds.), Proc. SIGCHI Conference on Human Factors in Computing Systems, ACM, Monterey, California, USA, 1992, pp. 219-226.

[8] A. Murata and H. Iwase, Usability of touch-panel interfaces for older adults, Human Factors 47 (2005), 766-776.

[9] S. Vetter, J. Bützler, N. Jochems and C.M. Schlick, Untersuchungen zum Fitts'schen Gesetz anhand eines großflächigen Touch-Displays, in M. Grandt \& A. Bauch, eds., Innovative Interaktionstechnologien für Mensch-Maschine-Schnittstellen, 52. Fachausschusssitzung Anthropotechnik der Deutschen Gesellschaft für Luft- und Raumfahrt Lilienthal-Oberth e.V., Bonn, 2010, pp. 147-158.

[10]T.G. Whisenand and H.H. Emurian, Analysis of cursor movements with a mouse, Computers in Human Behavior 15 (1999), 85-103

[11]H. Yang and $\mathrm{X} . \mathrm{Xu}$, Bias towards regular configuration in 2D pointing, in: Proc. of Conference on Human Factors in Computing Systems, ACM, CHI, 2010, pp. 1391-1400. 